\title{
Compensating for oblique ear-probe insertions in ear-canal reflectance measurements
}

\author{
Nørgaard, Kren Rahbek; Fernandez Grande, Efren; Laugesen, Søren
}

Published in:

Journal of the Acoustical Society of America

Link to article, DOI:

$10.1121 / 1.5111340$

Publication date:

2019

Document Version

Publisher's PDF, also known as Version of record

Link back to DTU Orbit

Citation (APA):

Nørgaard, K. R., Fernandez Grande, E., \& Laugesen, S. (2019). Compensating for oblique ear-probe insertions in ear-canal reflectance measurements. Journal of the Acoustical Society of America, 145(6), 3499-3509. https://doi.org/10.1121/1.5111340

\section{General rights}

Copyright and moral rights for the publications made accessible in the public portal are retained by the authors and/or other copyright owners and it is a condition of accessing publications that users recognise and abide by the legal requirements associated with these rights.

- Users may download and print one copy of any publication from the public portal for the purpose of private study or research.

- You may not further distribute the material or use it for any profit-making activity or commercial gain

- You may freely distribute the URL identifying the publication in the public portal 


\title{
Compensating for oblique ear-probe insertions in ear-canal reflectance measurements
}

\author{
Kren Rahbek Nørgaard ${ }^{a)}$ and Efren Fernandez-Grande \\ Acoustic Technology, Department of Electrical Engineering, Technical University of Denmark, Ørsteds Plads, \\ Building 352, Kongens Lyngby, DK-2800, Denmark
}

\author{
Søren Laugesen \\ Interacoustics Research Unit, Technical University of Denmark, Ørsteds Plads, Building 352, \\ Kongens Lyngby, DK-2800, Denmark
}

(Received 7 March 2019; revised 19 April 2019; accepted 22 May 2019; published online 18 June 2019)

\begin{abstract}
Measurements of the ear-canal reflectance using an ear probe require estimating the characteristic impedance of the ear canal in situ. However, an oblique insertion of the ear probe into a uniform waveguide prevents accurately estimating its characteristic impedance using existing time-domain methods. This is caused by the non-uniformity immediately in front of the ear probe when inserted at an oblique angle, resembling a short horn loading, and introduces errors into the ear-canal reflectance. This paper gives an overview of the influence of oblique ear-probe insertions and shows how they can be detected and quantified by estimating the characteristic impedance using multiple truncation frequencies, i.e., limiting the utilized frequency range. Additionally, a method is proposed to compensate for the effects on reflectance of an oblique ear-probe insertion into a uniform waveguide. The incident impedance of the horn loading is estimated, i.e., were the uniform waveguide anechoic, which replaces the characteristic impedance when calculating reflectance. The method can compensate for an oblique ear-probe insertion into a uniform occluded-ear simulator and decrease the dependency of reflectance on insertion depth in an ear canal. However, more research is required to further assess the method in ear canals. (C) 2019 Acoustical Society of America.
\end{abstract} https://doi.org/10.1121/1.5111340

[ICB]

Pages: $3499-3509$

\section{INTRODUCTION}

The ear-canal reflectance is a useful quantity for identifying conductive hearing disorders (Piskorski et al., 1999; Keefe et al., 2000; Feeney and Keefe, 2001; Keefe et al., 2012; Ellison et al., 2012; Merchant et al., 2015), calibrating stimulus levels in situ (Scheperle et al., 2008; McCreery et al., 2009; Lewis et al., 2009; Scheperle et al., 2011; Withnell et al., 2009; Souza et al., 2014), and estimating the sound pressure emitted from the ear (Charaziak and Shera, 2017). Measurements in the ear canal using an ear probe are affected by evanescent modes (Brass and Locke, 1997), and the ear-canal reflectance further depends on the characteristic impedance of the ear canal at the position of the ear probe. The characteristic impedance of a uniform waveguide is inversely proportional to its cross-sectional area, which inherently varies between different ears and with ear-probe insertion depth. Evanescent modes occur when an acoustic flow is injected into a waveguide through a narrow aperture and higher-order non-propagating modes are elicited. The effect of evanescent modes depends on the geometrical mismatch between the probe tube and the waveguide and can be approximated as an inertance in series with the plane-wave impedance in a one-dimensional propagation model (Keefe and Benade, 1981; Fletcher et al., 2005). Evanescent modes

\footnotetext{
a) Also at: Interacoustics A/S, Audiometer Allé 1, Middelfart, DK-5500, Denmark. Electronic mail: krng@interacoustics.com
}

and an incorrect characteristic impedance introduce errors into the ear-canal reflectance (Nørgaard et al., 2017a; Lewis, 2018), and must be compensated for and estimated, respectively, to obtain accurate measurements of the ear-canal reflectance, especially toward higher frequencies.

Rasetshwane and Neely (2011) estimated the characteristic impedance of the ear canal by minimizing the timedomain reflectance at time $t=0$. Nørgaard et al. (2017a) used a similar time-domain approach that also estimated an inertance (approximating the impedance of the evanescent modes) and subtracted it from the measured impedance. While Nørgaard et al. (2017a) showed that their method yields the plane-wave reflectance of a uniform occluded-ear simulator, they also noted that these time-domain methods for estimating the characteristic impedance depend on the acoustic waveguide being uniform at the position of the ear probe, a condition that is not satisfied when the ear probe is inserted into a uniform waveguide at an oblique angle using an ear tip.

Current widespread ear-canal impedance and reflectance measurement methods are based on a preliminary calibration of an ear probe to obtain its acoustic Thévenin-equivalent source parameters (Allen, 1986; Keefe et al., 1992; Voss and Allen, 1994). Available rubber ear tips (which are often favorable over foam ear tips for ear-canal impedance and reflectance measurements to barometrically and acoustically seal the ear probe to the ear canal) do not facilitate a placement of the ear probe in the ear canal in a way that aligns the 
orientation of the ear probe perpendicularly with the ear-canal walls. In addition, while the calibration procedure constitutes a controlled setup using straight uniform calibration waveguides, ear canals are inherently curved and non-uniform. Regardless of the type of ear tip, placing the ear probe at the position of a bend of the ear canal may result in a non-uniformity in front of the ear probe similar to that of an oblique insertion into a uniform waveguide, although it appears to be perpendicular as seen from the outside. In this paper, we assess and give an overview of the influence of oblique ear-probe insertions on the ear-canal reflectance when the characteristic impedance and evanescent-modes inertance are estimated in situ. In addition, we propose a method to detect such an oblique ear-probe insertion and compensate for its effects on reflectance in a uniform waveguide.

\section{BACKGROUND}

\section{A. Characteristic impedance}

For an acoustic plane wave propagating along a lossless uniform waveguide of cross-sectional area $A$, the ratio of sound pressure $P$ to volume flow $U$ is given by the characteristic impedance of the waveguide

$$
Z_{0}=\frac{P}{U}=\frac{\rho c}{A},
$$

with the air density $\rho$ and the speed of sound $c$. The frequency-independent purely real behavior of the characteristic impedance implies that, regardless of frequency, the sound pressure and volume flow are in phase with a constant ratio. This property gives the time-domain characteristic impedance $z_{0}(t)$ its desirable localized features,

$$
z_{0}(t)=\delta(t) Z_{0}
$$

where $\delta(\cdot)$ is the Dirac delta function, and enables estimating it in situ using the methods of Rasetshwane and Neely (2011) and Nørgaard et al. (2017a) for synthesized discrete time-domain signals using the inverse Fourier transform.

\section{B. Influence of insertion angle on ear-canal reflectance}

We assessed the influence of oblique ear-probe insertions on the ear-canal reflectance by inserting an ear probe into a uniform occluded-ear simulator using an ear tip at multiple angles relative to the cross-sectional plane of the ear simulator (such that $90^{\circ}$ corresponds to an ideal insertion). From the measured impedance $Z_{\text {meas }}$ [Eq. (29)], and the estimated characteristic impedance $\hat{Z}_{0}$ and inertance $\hat{L}$, Nørgaard et al. (2017a) calculated the reflectance as

$$
R^{\prime}=\frac{Z_{\text {meas }}-j \omega \hat{L}-\hat{Z}_{0}}{Z_{\text {meas }}-j \omega \hat{L}+\hat{Z}_{0}},
$$

such that the estimated inertance is subtracted from the measured impedance. $j$ is the unit imaginary number, $\omega$ the angular frequency, and the prime superscript denotes the reflectance calculated using this existing method. Figures 1(a) and 1(b) show the reflectance magnitudes $\left|R^{\prime}\right|$ [Eq. (3)] and the corresponding reflectance group delays $\tau_{\mathrm{g}}^{\prime}$ [Eq. (15)], respectively, and Fig. 1(c) shows $\hat{Z}_{0}$ and $\hat{L}$ for insertion angles from $90^{\circ}$ to $50^{\circ}$ (10 ${ }^{\circ}$ decrements). The measurements reveal a substantial influence of the insertion angle on the measured reflectance, especially when the angle falls below the region around $70^{\circ}$. In addition, $\hat{Z}_{0}$ exhibit an increasing deviation from the characteristic impedance of the occluded-ear simulator $Z_{0}$ [Eq. (1)] with decreasing insertion angle, and $\hat{L}$ a less conclusive behavior. Because the variation in the $R$ is large for frequencies as low as $2 \mathrm{kHz}$, it is reasonable to expect that it is caused mainly by changes in $\hat{Z}_{0}$ because the impedance of the estimated inertances $j \omega \hat{L}$ are low as those frequencies.

\section{Characteristics of an oblique insertion}

We examined the characteristics of an oblique ear-probe insertion and how it affects the coupling between the ear probe and waveguide by measuring the impedance of a uniform anechoic steel waveguide of radius $r=4 \mathrm{~mm}$ using perpendicular and oblique ear-probe insertions. The absence of reflections in the uniform waveguide enables to a large degree distinguishing the acoustic features of the ear-probe insertion from the features of the waveguide and evanescent modes. The difference in alignment and mechanical coupling is illustrated in Figs. 2(a) and 2(b) for the perpendicular and oblique insertions, respectively, of the ear probe into a transparent acrylic-glass waveguide, also of radius $r=4 \mathrm{~mm}$. We use $\perp$ and $\angle$ superscripts to denote measurements conducted with perpendicular and oblique ear-probe insertions, respectively. The chosen oblique ear-probe insertion represents the (a)

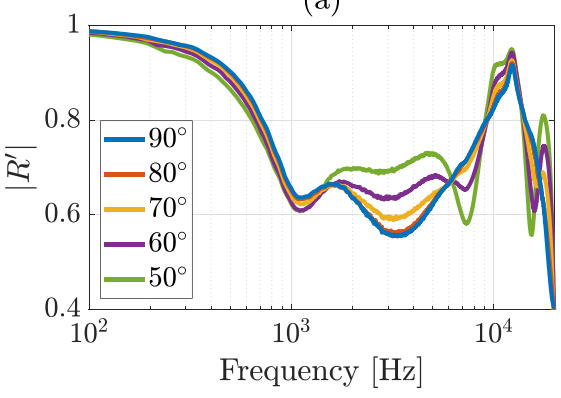

(b)

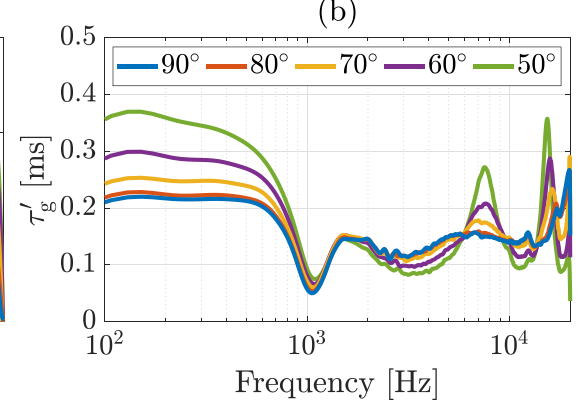

(c)

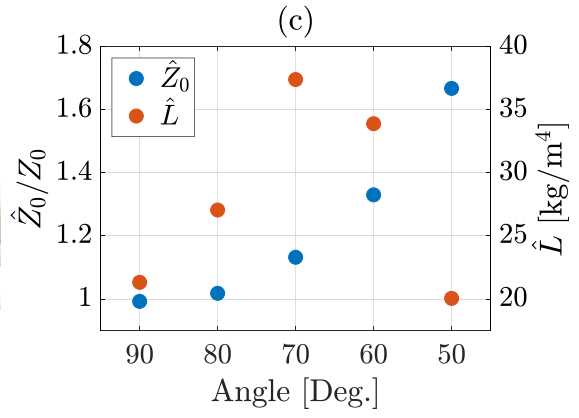

FIG. 1. (Color online) The (a) measured reflectance magnitudes $\left|R^{\prime}\right|$ [Eq. (3)], (b) corresponding reflectance group delays $\tau_{\mathrm{g}}^{\prime}$ [Eq. (15)], and (c) estimated normalized characteristic impedances $\hat{Z}_{0}$ and inertances $\hat{L}$ (Nørgaard et al., 2017a) in a uniform occluded-ear simulator with the ear probe inserted at the approximate angles relative to the cross-sectional plane. 


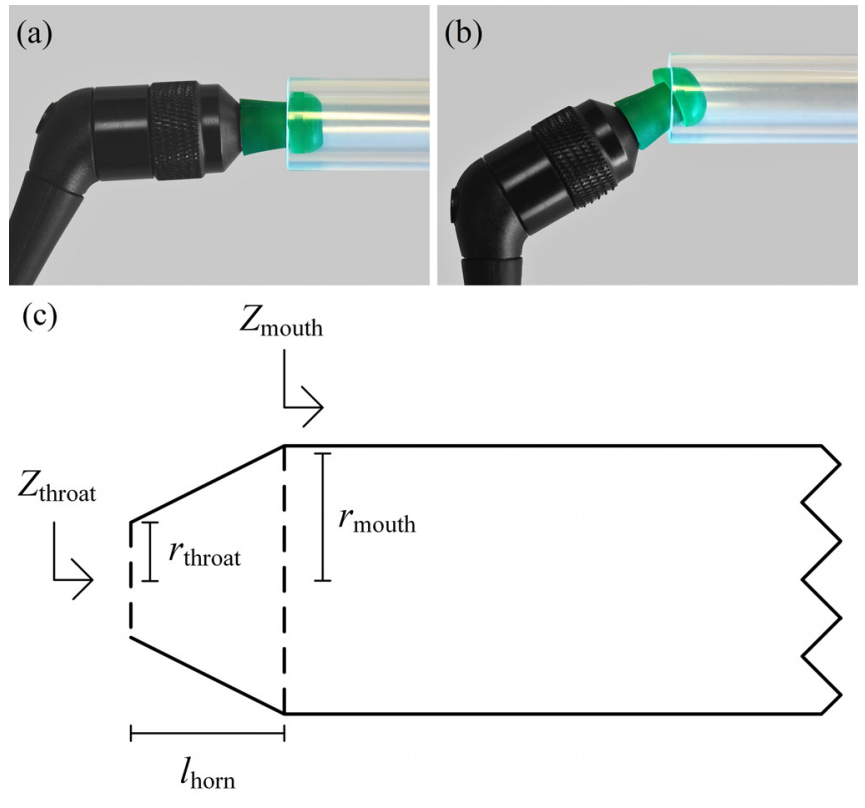

FIG. 2. (Color online) The (a) perpendicular $\perp$ and (b) oblique $\angle$ insertions of the ear probe into an acrylic-glass waveguide of radius $r=4 \mathrm{~mm}$, and (c) a cross-sectional illustration of the conical horn segment approximating an oblique ear-probe insertion, including its impedance and dimensional variables, terminated by a uniform waveguide.

worst-case insertion angle that could be obtained in a uniform waveguide without introducing a leak in the probe fit $\left(\sim 50^{\circ}\right)$. Figure $3(\mathrm{a})$ shows the real $\operatorname{Re}\{\cdot\}$ and imaginary $\operatorname{Im}\{\cdot\}$ parts of the measured incident impedances of the anechoic waveguide $Z_{\text {meas }, 0}^{\perp}$ and $Z_{\text {meas }, 0}^{\perp}$ [Eq. (29)]. We use the term incident impedance to denote the impedance that one measures in a uniform anechoic waveguide including the effects of the coupling between the ear probe and waveguide, i.e., including an oblique insertion. As expected, $Z_{\text {meas }, 0}^{\perp} \approx Z_{0}+j \omega L_{\mathrm{em}}$, so that the evanescent-modes inertance $L_{\mathrm{em}}$ can be directly identified. Conversely, $\operatorname{Re}\left\{Z_{\text {meas }, 0}^{\perp}\right\}$ increases with frequency, diverging from $Z_{0}$, and the inertance in $\operatorname{Im}\left\{Z_{\text {meas }, 0}^{\llcorner}\right\}$has increased.

Consider a conical horn segment of throat radius $r_{\text {throat }}$ $=2.5 \mathrm{~mm}$, mouth radius $r_{\text {mouth }}=4 \mathrm{~mm}$, and horn length $l_{\text {horn }}=4 \mathrm{~mm}$. We calculated the analytical plane-wave incident throat impedance $Z_{\text {throat }, 0}$ of the horn segment, i.e., terminated by the characteristic impedance at the mouth $Z_{0 \text {,mouth }}=\rho c / \pi r_{\text {mouth }}^{2}$, using a lossless conical transmission-

line segment and the corresponding transfer-matrix elements $a_{i j}$ (Caussé et al., 1984; Benade, 1988),

$$
Z_{\text {throat }, 0}=\frac{a_{11} Z_{0, \text { mouth }}+a_{12}}{a_{21} Z_{0, \text { mouth }}+a_{22}} .
$$

We denote plane-wave impedances as such to indicate a one-dimensional sound field and an absence of higher-order modes, despite the physical waves inside a conical horn segment being spherical. We chose $r_{\text {mouth }}$ to match that of the anechoic waveguide, and $r_{\text {throat }}$ and $l_{\text {horn }}$ by trial and error to approximate the measurements in Fig. 3(a). Figure 2(c) shows an illustration of a conical horn segment, including impedance and dimensional variables at the mouth and throat, terminated by a uniform waveguide. Figure 3(b) shows the normalized real and imaginary parts of the analytical plane-wave incident throat impedance of the conical horn segment $Z_{\text {throat }, 0}$ [Eq. (4)]. $\operatorname{Re}\left\{Z_{\text {throat }, 0}\right\}$ is similar to $\operatorname{Re}\left\{Z_{\text {meas }, 0}^{L}\right\}$ in Fig. 3(a), suggesting that the conical horn segment approximates this worst-case oblique ear-probe insertion up to $20 \mathrm{kHz}$. In addition, the horn loading contributes with an approximate inertance $L_{\text {horn }}$ to $\operatorname{Im}\left\{Z_{\text {throat }, 0}\right\}$ $\approx \omega L_{\text {horn }}$. When the wavelength is much longer than the horn length $\lambda \gg l_{\text {horn }}$, a similar level of accuracy in approximating an oblique ear-probe insertion up to $20 \mathrm{kHz}$ can be obtained using different horn shapes, e.g., exponential, but the conical horn was chosen for this example since it is the simplest geometrical shape of a horn and straightforward to model analytically using a single segment. In addition to deviations in the estimated characteristic impedance $\hat{Z}_{0}$ and inertance $\hat{L}$, the variation in reflectance in Fig. 1 can therefore also be attributed to the presence of this horn loading.

\section{Estimated characteristic impedance and inertance}

Nørgaard et al. (2017a) used the Hilbert transform $\mathcal{H}[\cdot]$ to calculate the impedance estimation error

$$
\epsilon_{Z}=Z-Z_{0}-\mathcal{H}[\operatorname{Im}\{Z\}]-j \mathcal{H}^{-1}[\operatorname{Re}\{Z\}]
$$

of an impedance $Z$. From the measured impedance $Z_{\text {meas }}$, they estimated the characteristic impedance $\hat{Z}_{0}$, and inertance $\hat{L}$ by minimizing the real and imaginary parts of the corresponding impedance estimation error $\epsilon_{Z_{\text {meas }}}$, respectively. Such time-domain methods essentially separate the physical features of the acoustic load at the position of the

(b)

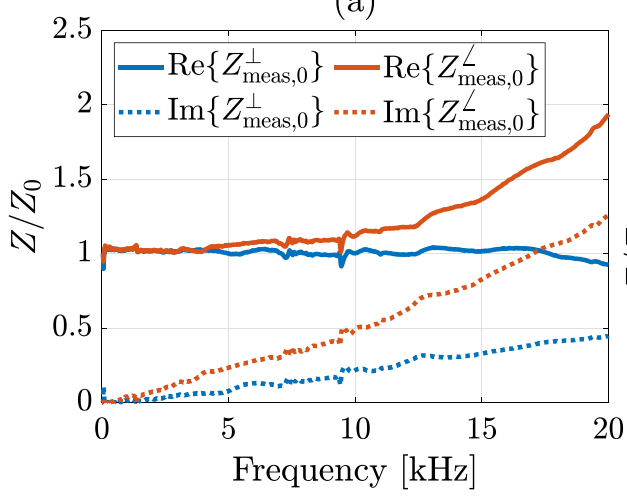

FIG. 3. (Color online) The normalized real and imaginary parts of the (a) measured incident impedances in the anechoic waveguide $Z_{\text {meas. } 0}^{\perp}$ and $Z_{\text {meas. } 0}^{\perp}$ [Eq. (29)] using the perpendicular and oblique ear-probe insertions, respectively, (b) analytical plane-wave incident throat impedance $Z_{\text {throat }, 0}$ [Eq. (4)] of the conical horn segment, and the corresponding imaginary impedance estimation error $\operatorname{Im}\left\{\epsilon_{Z_{\text {throat }, 0}}\right\}$ [Eq. (5)]. Note the equivalent normalization $Z_{0}=Z_{0, \text { mouth }}$. 
ear probe from the features of the waveguide, to the degree allowed by the finite sampling rate in the synthesized timedomain transfer functions, and the termination. Due to the finite speed of sound, $\hat{Z}_{0}$ and $\hat{L}$ estimated from finite measurement bandwidths therefore include some spatial information about the waveguide in front of the ear probe. When the wavelength of the maximum measurement frequency is much longer than the horn length $\lambda \gg l_{\text {horn }}$, then $\hat{Z}_{0}$ and $\hat{L}$ can be represented in terms of the measured incident impedance $Z_{\text {meas, } 0}$, i.e., with an anechoic termination, and the corresponding impedance estimation error $\epsilon_{Z_{\text {meas }, 0}}$.

The estimated characteristic impedance $\hat{Z}_{0}$ represents the averaged $\langle\cdot\rangle$ real part of the measured incident impedance $Z_{\text {meas }, 0}$,

$$
\hat{Z}_{0}=\left\langle\operatorname{Re}\left\{Z_{\text {meas }, 0}\right\}\right\rangle \text {. }
$$

Consequently, due to the behavior in $\operatorname{Re}\left\{Z_{\text {meas }, 0}^{\perp}\right\}$ [see Fig. 3(a)], $\hat{Z}_{0}$ no longer represents the characteristic impedance of the waveguide $Z_{0}$, which explains the increasing deviation from $Z_{0}$ in Fig. 1(c) with decreasing insertion angle. Note that, if a frequency-domain window is applied to the reflectance (e.g., Rasetshwane and Neely, 2011), the average in Eq. (6) is weighted accordingly.

Similarly, the estimated inertance $\hat{L}$ represents the quantity that minimizes $\operatorname{Im}\left\{\epsilon_{Z_{\text {meas }, 0}}\right\}$ when subtracted from $Z_{\text {meas }, 0}$. Assuming a frequency-linear behavior in $\operatorname{Im}\left\{\epsilon_{Z_{\text {meas }, 0}}\right\}$,

$$
\hat{L}=\left\langle\operatorname{Im}\left\{\epsilon_{Z_{\text {meas }, 0}}\right\} / \omega\right\rangle \text {. }
$$

To exemplify the consequence for $\hat{L}$, Fig. 3(b) further shows the imaginary part of the impedance estimation error $\epsilon_{Z_{\text {throat } .0}}$ [Eq. (5)] resulting from the analytical plane-wave incident throat impedance $Z_{\text {throat }, 0}$ [Eq. (4)]. Although $Z_{\text {throat }, 0}$ is a plane-wave quantity, notice the approximate frequencylinear behavior in $\operatorname{Im}\left\{\epsilon_{Z_{\text {throat }, 0}}\right\} \neq \operatorname{Im}\left\{Z_{\text {throat }, 0}\right\} \neq 0$. This is a result of the behavior in $\operatorname{Re}\left\{Z_{\text {throat }, 0}\right\}$ when applying the Hilbert transform over a finite frequency range in Eq. (5) and implies that the method of Nørgaard et al. (2017a) can neither reveal the inertances originating from evanescent-modes $L_{\mathrm{em}}$, the horn loading $L_{\mathrm{horn}}$, nor the total inertance

$$
L_{0}=L_{\mathrm{em}}+L_{\mathrm{horn}},
$$

given an oblique ear-probe insertion. This does not fully explain the behavior of $\hat{L}$ in Fig. 1(c) because $L_{\mathrm{em}}$ depends on the geometrical mismatch between the ear probe and waveguide (Keefe and Benade, 1981; Fletcher et al., 2005), i.e., the throat radius $r_{\text {throat }}$ in the equivalent conical-horn model. While $L_{\text {horn }}$ consistently increases when the insertion angle decreases, $L_{\mathrm{em}}$ exhibits a completely different behavior and vanishes for a certain radius [see Fig. 3 in Fletcher et al. (2005)].

\section{THEORY}

Consider an arbitrary non-uniform horn segment in front of a uniform waveguide such as shown for a conical segment in Fig. 2(c). Given the plane-wave throat impedance $Z_{\text {throat }}$, the plane-wave mouth impedance $Z_{\text {mouth }}$ can be calculated using the one-dimensional transfer-matrix elements $a_{i j}$ of the horn segment,

$$
Z_{\text {mouth }}=\frac{a_{22} Z_{\text {throat }}-a_{12}}{a_{11}-a_{21} Z_{\text {throat }}} .
$$

The desired reflectance of the uniform waveguide at the mouth is given by

$$
R_{\text {mouth }}=\frac{Z_{\text {mouth }}-Z_{0, \text { mouth }}}{Z_{\text {mouth }}+Z_{0, \text { mouth }}} .
$$

Combining Eqs. (9) and (10), and rearranging yields

$$
R_{\text {mouth }}=\frac{Z_{\text {throat }}-\frac{a_{11} Z_{0, \text { mouth }}+a_{12}}{a_{21} Z_{0, \text { mouth }}+a_{22}}}{Z_{\text {throat }}+\frac{a_{11} Z_{0, \text { mouth }}-a_{12}}{-a_{21} Z_{0, \text { mouth }}+a_{22}}} \cdot \frac{a_{21} Z_{0, \text { mouth }}+a_{22}}{-a_{21} Z_{0, \text { mouth }}+a_{22}} .
$$

For a lossless transmission line, $a_{11}, a_{22}$, and $Z_{0, \text { mouth }}$ are real numbers, and $a_{12}$ and $a_{21}$ are imaginary numbers. With the plane-wave incident throat impedance $Z_{\text {throat }, 0}$ as given in Eq. (4) and the plane-wave incident transfer impedance of the horn segment $Z_{\text {trans }, 0}$, i.e., the ratio of sound pressure at the mouth $P_{\text {mouth }}$ to volume flow injected at the throat $U_{\text {throat }}$, of the horn segment terminated by $Z_{0, \text { mouth }}$,

$$
Z_{\text {trans }, 0}=\frac{P_{\text {mouth }}}{U_{\text {throat }}}=\frac{Z_{0, \text { mouth }}}{a_{21} Z_{0, \text { mouth }}+a_{22}},
$$

Eq. (11) can be written as

$$
R_{\text {mouth }}=\frac{Z_{\text {throat }}-Z_{\text {throat }, 0}}{Z_{\text {throat }}+Z_{\text {throat }, 0}^{*}} \cdot \frac{Z_{\text {trans }, 0}^{*}}{Z_{\text {trans }, 0}} .
$$

The asterisk superscript denotes the complex conjugate.

\section{METHODS}

\section{A. Compensating for an oblique ear-probe insertion}

For any complex number $z$,

$$
z^{*} / z=e^{-j 2 \angle z}
$$

with the phase operator $\angle$. Given the plane-wave throat impedance $Z_{\text {throat }}$ and plane-wave incident throat impedance $Z_{\text {throat }, 0}$ in Eq. (13), the horn loading merely contributes with a frequency-dependent phase shift $e^{-j 2 \angle Z_{\text {trans }, 0}}$ and the effects on the reflectance magnitude at the mouth $\left|R_{\text {mouth }}\right|$ can be compensated for. If an exact compensation of the reflectance group delay,

$$
\tau_{\mathrm{g}}=-\frac{\mathrm{d} \angle R}{\mathrm{~d} \omega},
$$

is needed, it requires estimating the plane-wave incident transfer-impedance phase shift $e^{-j 2 \angle Z_{\text {trans }, 0}}$ or the transmissionline matrix elements $a_{i j}$. 
In a practical measurement scenario using an ear probe, any measured impedance $Z_{\text {meas }}$ and incident impedance $Z_{\text {meas }, 0}$ are affected by evanescent modes, assumed to comprise the series impedance of an evanescent-modes inertance $j \omega L_{\mathrm{em}}$ to the corresponding plane-wave quantities. If the evanescent modes have decayed before being reflected at the termination of the uniform waveguide, $L_{\mathrm{em}}$ is identical for $Z_{\text {meas }}$ and $Z_{\text {meas }, 0}$,

$$
\begin{aligned}
& Z_{\text {meas }} \simeq Z_{\text {throat }}+j \omega L_{\mathrm{em}}, \\
& Z_{\text {meas }, 0} \simeq Z_{\text {throat }, 0}+j \omega L_{\mathrm{em}},
\end{aligned}
$$

and vanishes from Eq. (13) when substituting $Z_{\text {meas }}$ and $Z_{\text {meas }, 0}$ in place of $Z_{\text {throat }}$ and $Z_{\text {throat }, 0}$, respectively, due to the complex conjugate in the denominator.

We chose to disregard the frequency-dependent phase shift $e^{-j 2 \angle Z_{\text {trans, } 0}}$ due to the horn loading in Eq. (13) and calculate the reflectance as

$$
R=\frac{Z_{\text {meas }}-Z_{\text {meas }, 0}}{Z_{\text {meas }}+Z_{\text {meas }, 0}^{*}} .
$$

The formulation in Eq. (18) is particularly advantageous because $Z_{\text {meas }}$ and $Z_{\text {meas }, 0}$ both represent measured quantities, i.e., affected by evanescent modes, thus eliminating the need to uniquely identify the inertances originating from evanescent modes $L_{\mathrm{em}}$ and the horn loading $L_{\mathrm{horn}}$ as would be required using Eq. (9). In addition, Eq. (18) does not assume any geometrical features of the horn loading, such as it being conical. When $r_{\text {throat }}=r_{\text {mouth }}$, then $Z_{\text {meas }, 0} \simeq Z_{0}+j \omega L_{\text {em }}$ and Eqs. (3) and (18) are equivalent $\left(\angle Z_{\text {trans }, 0}=-\omega l_{\text {horn }} / c\right)$. Naturally, $Z_{\text {meas } 0}$ is unknown for an impedance measurement in a uniform waveguide of finite length and needs to be estimated.

\section{B. Detecting an oblique ear-probe insertion}

We propose a method to detect an oblique ear-probe insertion into a uniform waveguide that limits the frequency range utilized for estimating the characteristic impedance $\hat{Z}_{0}$ and inertance $\hat{L}$ using the method of Nørgaard et al. (2017a) to some variable truncation frequency $\omega_{\mathrm{t}}$. From Eq. (6), the estimated characteristic impedance as a function of the truncation frequency $\hat{Z}_{0}\left(\omega_{\mathrm{t}}\right)$ represents the cumulative average

$$
\hat{Z}_{0}\left(\omega_{\mathrm{t}}\right)=\frac{1}{\omega_{\mathrm{t}}} \int_{0}^{\omega_{\mathrm{t}}} \operatorname{Re}\left\{Z_{\text {meas }, 0}\right\} \mathrm{d} \omega .
$$

By estimating $\hat{Z}_{0}\left(\omega_{\mathrm{t}}\right)$ using multiple truncation frequencies, any variation in $\hat{Z}_{0}\left(\omega_{\mathrm{t}}\right)$ indicates that the acoustic load is non-uniform at the position of the ear probe and that the estimated characteristic impedances may be invalid. In a uniform waveguide, we can assert that variations in $\hat{Z}_{0}\left(\omega_{\mathrm{t}}\right)$ are caused by an oblique ear-probe insertion.

In waveguides of finite length, such as an ear canal, the method of Nørgaard et al. (2017a) relies on resampling the synthesized time-domain reflectance by truncating the frequency spectrum, such that differentiability is restored at this truncation frequency $\omega_{\mathrm{t}}$, and simply utilizes the largestpossible $\omega_{\mathrm{t}}$. This is the frequency at which the Hermitian- symmetric frequency spectrum is replicated when numerically evaluating the Hilbert transform in Eq. (5). Thus, a finite number $N$ of truncation frequencies $\omega_{\mathrm{t}}$ exist for a given maximum measurement frequency $\omega_{\mathrm{m}}$ and total length of the acoustic load $l$,

$$
N \approx\left\lfloor\frac{2 l \omega_{\mathrm{m}}}{\pi c}\right\rfloor,
$$

where $\lfloor\cdot\rfloor$ denotes flooring to nearest integer. This maximum frequency $\omega_{\mathrm{m}}$ is different from the Nyquist frequency of the data-acquisition system due to anti-aliasing filters and potential limitations in the acoustic Thévenin-equivalent calibration of the ear probe. Thus, $N$ is also the number of truncation frequencies $\omega_{\mathrm{t}}$ which can be used to estimate $\hat{Z}_{0}$ and $\hat{L}$ using the method of Nørgaard et al. (2017a). For plane waves in a rigidly terminated lossless uniform waveguide, Eq. (20) is exact, but evanescent modes, non-uniformities, and a finite termination impedance can translate these points in frequency although the physical length remains unchanged.

\section{Estimating the incident impedance}

To estimate the incident impedance $\hat{Z}_{\text {meas }, 0}$ for the case that the wavelength is much longer than the horn length $\lambda \gg l_{\text {horn }}$, we fit simple polynomials to the discrete sets of estimated characteristic impedances $\hat{Z}_{0}\left(\omega_{\mathrm{t}}\right)$ and inertances $\hat{L}\left(\omega_{\mathrm{t}}\right)$ using all possible truncation frequencies $\omega_{\mathrm{t}}$. To accommodate for the limited number of truncation frequencies [Eq. (20)], each polynomial consists of two parameters, an ordinate intercept (essentially representing the lumped-element behavior of the horn loading) and a frequency-dependent constant, to obtain a continuousfrequency representation of the estimated characteristic impedances and inertances, from which $\hat{Z}_{\text {meas }, 0}$ can be extracted. Although $\hat{Z}_{\text {meas }, 0}$ is an estimated quantity, we retain its subscript because it represents the quantity as would be measured using an identical ear-probe insertion into an anechoic uniform waveguide.

Figure 4(a) shows initially the normalized real part of the incident impedance $Z_{\text {meas }, 0}$ (for this example calculated analytically from $Z_{\text {throat }, 0}$ [Eq. (4)] and Eq. (17) of the conical horn segment from Sec. IIC), and its cumulative average $\hat{Z}_{0}\left(\omega_{\mathrm{t}}\right)$ [Eq. (19)]. We represent the behavior in $\hat{Z}_{0}\left(\omega_{\mathrm{t}}\right)$ as a second-order polynomial,

$$
\hat{Z}_{0}\left(\omega_{\mathrm{t}}\right)=\hat{Z}_{0}^{\prime \prime} \omega_{\mathrm{t}}^{2}+\hat{Z}_{0, \mathrm{ec}}
$$

with concavity $\hat{Z}_{0}^{\prime \prime}$ and ordinate intercept $\hat{Z}_{0, \mathrm{ec}}$, the latter representing an estimate of the characteristic impedance of the ear canal. Combining Eqs. (19) and (21), and solving for $\operatorname{Re}\left\{Z_{\text {meas }, 0}\right\}$, we estimate the real part of the incident impedance,

$$
\operatorname{Re}\left\{\hat{Z}_{\text {meas }, 0}\right\}=3 \hat{Z}_{0}^{\prime \prime} \omega^{2}+\hat{Z}_{0, \mathrm{ec}}
$$

Figure 4(a) further shows the second-order polynomial $\hat{Z}_{0}^{\prime \prime} \omega_{\mathrm{t}}^{2}+\hat{Z}_{0, \text { ec }}$ [Eq. (21)], fitted to $\hat{Z}_{0}\left(\omega_{\mathrm{t}}\right)$ using least squares, and the real part of the estimated incident impedance 
(a)

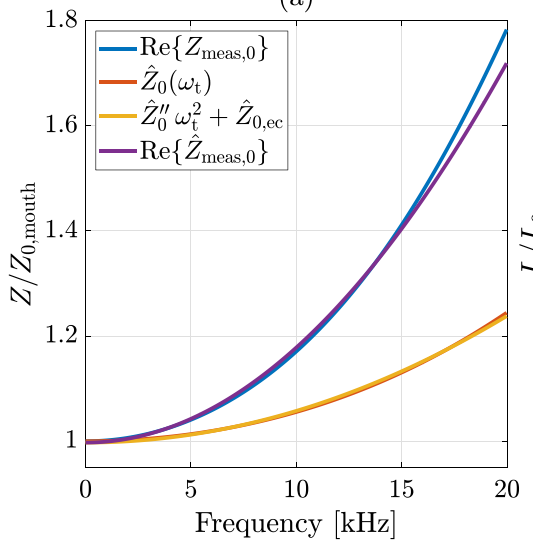

(b)

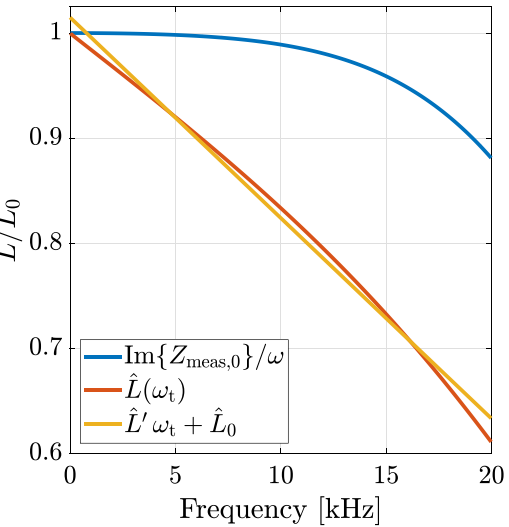

FIG. 4. (Color online) (a) The normalized real part of the analytical incident impedance $\operatorname{Re}\left\{Z_{\text {meas }, 0}\right\}$ [Eqs. (4) and (17)] of the conical horn segment of Sec. IIC, its cumulative average $\hat{Z}_{0}\left(\omega_{\mathrm{t}}\right)$ [Eq. (19)], second-order polynomial $\hat{Z}_{0}^{\prime \prime} \omega_{\mathrm{t}}^{2}+\hat{Z}_{0, \text { ec }}$ [Eq. (21)], fitted to $\hat{Z}_{0}\left(\omega_{\mathrm{t}}\right)$, and real part of the estimated incident impedance $\operatorname{Re}\left\{\hat{Z}_{\text {meas }, 0}\right\}$ [Eq. (22)]. (b) The frequency-normalized $\operatorname{Im}\left\{Z_{\text {meas. } 0}\right\}$, the estimated inertances with different truncation frequencies $\hat{L}\left(\omega_{\mathrm{t}}\right)$ [Eq. (7)], and the first-order polynomial $\hat{L}^{\prime} \omega_{\mathrm{t}}+\hat{L}_{0}$ [Eq. (25)], fitted to $\hat{L}\left(\omega_{\mathrm{t}}\right)$, normalized with respect to the true size of the inertance $L_{0}$ [Eq. (8)].
$\operatorname{Re}\left\{\hat{Z}_{\text {meas }, 0}\right\}$ [Eq. (22)]. The representation as a second-order polynomial provides reasonable accuracy within the fitted frequency range and largely captures the features in $\operatorname{Re}\left\{Z_{\text {meas }, 0}\right\}$.

We approximate the imaginary part of $\hat{Z}_{\text {meas.0 }}$ as the estimated total inertance $\hat{L}_{0}$ [see Eq. (8)], defined in the limit of low frequencies,

$$
\operatorname{Im}\left\{\hat{Z}_{\text {meas }, 0}\right\}=\omega \hat{L}_{0}=\omega \lim _{\omega \rightarrow 0} \operatorname{Im}\left\{Z_{\text {meas }, 0}\right\} / \omega .
$$

When $\operatorname{Re}\left\{\hat{Z}_{\text {meas }, 0}\right\}$ behaves similar to the second-order polynomial in Eq. (22), the estimated inertance in Eq. (7) as a function of the truncation frequency $\hat{L}\left(\omega_{\mathrm{t}}\right)$,

$$
\lim _{\omega_{\mathrm{t}} \rightarrow 0} \hat{L}\left(\omega_{\mathrm{t}}\right)=L_{0}
$$

because the Hilbert transform of a constant is zero. Figure 4(b) shows initially the imaginary part of the frequencynormalized analytical incident impedance $\operatorname{Im}\left\{Z_{\text {meas }, 0}\right\}$ [Eq. (4)] of the same exemplary conical horn segment and the estimated inertances with different truncation frequencies $\hat{L}\left(\omega_{\mathrm{t}}\right)$ [Eq. (7)]. We represent the behavior in $\hat{L}\left(\omega_{\mathrm{t}}\right)$ as a first-order polynomial to obtain the ordinate intercept $\hat{L}_{0}$,

$$
\hat{L}\left(\omega_{\mathrm{t}}\right)=\hat{L}^{\prime} \omega_{\mathrm{t}}+\hat{L}_{0}
$$

and the slope $\hat{L}^{\prime}$ of the estimated inertance with truncation frequency. Figure 4(b) further shows the first-order polynomial $\hat{L}^{\prime} \omega_{\mathrm{t}}+\hat{L_{0}}$ [Eq. (25)] fitted to $\hat{L}\left(\omega_{\mathrm{t}}\right)$ using least squares, resulting in an accurate estimate of $L_{0}$.

\section{Summary of the proposed method}

The proposed method of detecting and compensating for the effect of an oblique ear-probe insertion on reflectance in a uniform waveguide can be summarized by the following steps:

(1) Estimate the characteristic impedances $\hat{Z}_{0}\left(\omega_{\mathrm{t}}\right)$ and the inertances $\hat{L}\left(\omega_{\mathrm{t}}\right)$ using the method of Nørgaard et al. (2017a) at all possible truncation frequencies $\omega_{\mathrm{t}}$.

(2) Variations in $\hat{Z}_{0}\left(\omega_{\mathrm{t}}\right)$ signify an oblique ear-probe insertion. For a perpendicular insertion, indicated by a constant $\hat{Z}_{0}\left(\omega_{\mathrm{t}}\right)$, no further compensation is required and the reflectance can be calculated using the existing method [Eq. (3), Nørgaard et al., 2017a].

(3) If an oblique ear-probe insertion is detected, fit the second-order polynomial $\hat{Z}_{0}^{\prime \prime} \omega_{\mathrm{t}}^{2}+\hat{Z}_{0, \mathrm{ec}}$ to $\hat{Z}_{0}\left(\omega_{\mathrm{t}}\right)$, the first-order polynomial $\hat{L}^{\prime} \omega_{\mathrm{t}}+\hat{L}_{0}$ to $\hat{L}\left(\omega_{\mathrm{t}}\right)$, and estimate the incident impedance,

$$
\hat{Z}_{\text {meas }, 0}=3 \hat{Z}_{0}^{\prime \prime} \omega^{2}+\hat{Z}_{0, \mathrm{ec}}+j \omega \hat{L}_{0}
$$

(4) The effect of the oblique ear-probe insertion on reflectance can now be compensated for by calculating the reflectance $R$ from the measured impedance $Z_{\text {meas }}$ [Eq. (29)],

$$
R=\frac{Z_{\text {meas }}-\hat{Z}_{\text {meas }, 0}}{Z_{\text {meas }}+\hat{Z}_{\text {meas }, 0}^{*}} .
$$

The plane-wave impedance at the position of the ear probe, unaffected by the horn loading $\hat{Z}_{\mathrm{pw}}$ can be estimated from

$$
\hat{Z}_{\mathrm{pw}}=\hat{Z}_{0, \mathrm{ec}} \frac{1+R}{1-R} .
$$

\section{E. Equipment and measurements}

The measurements reported in this study were carried out using a FireFace UC sound card (RME Audio, Haimhausen, Germany) controlled through custom-written MATLAB (The MathWorks, Inc., Natick, MA) software and the third-party utility PLAYREC (Humphrey, 2014). Reported reflectance group delays $\tau_{\mathrm{g}}$ [Eq. (15)] were convolved with a 19-point Blackman window to reduce noise. A Titan-based ear probe (Interacoustics A/S, Middelfart, Denmark) was used, but modified to improve the high-frequency performance and reduce internal crosstalk.

Probe pressures were measured by supplying a frequencyequalized wideband chirp to the ear probe to provide a flat probe pressure in an anechoic waveguide of radius similar to an adult ear canal. The chirp was played back in the ear probe in phase-locked 2048-sample blocks at a sampling rate of 44.1 $\mathrm{kHz}$ which were each recorded using the ear-probe microphone and averaged to reduce noise in the measurements. Prior to the 
measurements, the ear probe was calibrated to obtain its acoustic Thévenin-equivalent source parameters, the source pressure $P_{\mathrm{s}}$ and source impedance $Z_{\mathrm{s}}$, and enable the measurement of acoustic impedance using the calibration method described by Nørgaard et al. (2017b). The cylindrical calibration waveguides were of lengths $l=1.2,1.45,1.75$, and $2 \mathrm{~cm}$, and radius $r=2 \mathrm{~mm}$. Note that when the source parameters are free from parallel components, impedance measurements are independent of the mismatch in radius between the calibration waveguides and the acoustic load (Nørgaard et al., 2017b, 2018). The maximum calibration frequency, and thereby maximum impedance measurement frequency, was $\omega_{\mathrm{m}} / 2 \pi=20 \mathrm{kHz}$. Measured impedances $Z_{\text {meas }}$ were derived from the measured probe pressure $P_{\text {meas }}$ and the Thévenin-equivalent source parameters,

$$
Z_{\text {meas }}=Z_{\mathrm{s}} \frac{P_{\text {meas }}}{P_{\mathrm{s}}-P_{\text {meas }}} .
$$

The anechoic waveguide utilized in Sec. II C consisted of a coiled steel tube of radius $r=4 \mathrm{~mm}$ and length $l=6 \mathrm{~m}$ such that the incident and reflected probe-pressure waveforms could be separated in time, effectively causing the waveguide to appear anechoic. This was achieved by increasing the length of the phase-locked blocks to 8192 samples, enough for reflections to decay before the next block, and truncating the length down to 2048 samples to eliminate these reflections. In addition, a Type 4157 occluded-ear simulator (Brüel \& Kjær Sound \& Vibration A/S, Nærum, Denmark), typically designated a 711 coupler, was used to evaluate the proposed method in a load similar to the human ear canal. A 3D-printed part was attached to the ear simulator that uniformly extended its length, such that it more accurately resembled a typical ear-probe insertion into an ear canal and allowed for a similar number of truncation frequencies [Eq. (20)]. The ear simulator was of dimensions, radius $r=3.75 \mathrm{~mm}$ and length $l=2.75 \mathrm{~cm}$ (minus insertion depth). The ear-probe was inserted into the waveguides using a standard, mushroom-shaped, green, 9-mm rubber ear tip (Sanibel Supply, Middelfart, Denmark). Finally, measurements in a single ear canal (right ear of the first author) were carried out using three different insertion depths: deep, medium, and shallow, achieved using three ear-tip sizes, $9 \mathrm{~mm}$ green, $10 \mathrm{~mm}$ red, and $11 \mathrm{~mm}$ blue, respectively, of the same type as described above. We chose this approach because it seemed that variation in the apparent obliqueness of the ear-probe insertion could partly be caused by variations in curvature along the length of the ear canal. The different ear-tip sizes were chosen to achieve the different insertion depths with a thorough insertion of the ear probe into the ear canal. The measurements were approved by the Science-Ethics Committee for the Capital Region of Denmark.

\section{RESULTS}

\section{A. Occluded-ear simulator}

To assess the capability of the proposed method under controlled conditions, we measured the impedances of the occluded-ear simulator $Z_{\text {meas }}^{\perp}$ and $Z_{\text {meas }}^{\perp}$ [Eq. (29)] using perpendicular and oblique $\left(\sim 50^{\circ}\right)$ ear-probe insertions, respectively [see Fig. 2]. The characteristic impedances $\hat{Z}_{0}\left(\omega_{\mathrm{t}}\right)$ and inertances $\hat{L}\left(\omega_{\mathrm{t}}\right)$ were estimated (Nørgaard et al., 2017a) for six different truncation frequencies $\omega_{\mathrm{t}}$. Figure 5(a) shows $\hat{Z}_{0}\left(\omega_{\mathrm{t}}\right)$ and the fitted second-order polynomial $\hat{Z}_{0}^{\prime \prime} \omega_{\mathrm{t}}^{2}+\hat{Z}_{0, \mathrm{ec}}$ [Eq. (21)], normalized by the known characteristic impedance $Z_{0}$ [Eq. (1)], for the perpendicular and oblique insertions. $Z_{0}$ and the estimated quantities $\hat{Z}_{0}^{\prime \prime}$ and $\hat{Z}_{0, \text { ec }}$ are initially listed in Table I. The truncation frequencies were all in the range of $3-20 \mathrm{kHz}$ and the individual frequencies can be approximately identified on the $x$ axis from the dots in Figs. 5(a) and 5(b), and were slightly different for the two insertions. For the perpendicular insertion, $\hat{Z}_{0}\left(\omega_{\mathrm{t}}\right)$ is largely independent of $\omega_{\mathrm{t}}$, whereas the oblique insertion resulted in and was confirmed by significantly increasing $\hat{Z}_{0}\left(\omega_{\mathrm{t}}\right)$ with a similar pattern to that in Fig. 4. Note that the estimated ear-canal characteristic impedances $\hat{Z}_{0, \mathrm{ec}}$ align well with $Z_{0}$ in both cases. Figure 5(b) shows $\hat{L}\left(\omega_{\mathrm{t}}\right)$ and the fitted first-order polynomial $\hat{L}^{\prime} \omega_{\mathrm{t}}+\hat{L}_{0}$ [Eq. (25)] for the perpendicular and oblique insertions. The estimated quantities $\hat{L}^{\prime}$ and $\hat{L}_{0}$ are further listed in Table I. For the perpendicular insertion, although there is some variation between the different $\hat{L}\left(\omega_{\mathrm{t}}\right)$, the slope of the fitted line is negligible, i.e., $\hat{L}\left(\omega_{\mathrm{t}}\right) \approx \hat{L}_{0}$. For the oblique insertion, $\hat{L}\left(\omega_{\mathrm{t}}\right)$ varied substantially with $\omega_{\mathrm{t}}$ and resulted in a significantly larger $\hat{L}_{0}$.

We calculated the reflectances $R^{\perp}$ and $R^{\perp}$ [Eq. (27)] using the estimated incident impedances $\hat{Z}_{\text {meas }, 0}^{\perp}$ and $\hat{Z}_{\text {meas }, 0}^{\perp}$ [Eq. (26)], respectively, based on $\hat{Z}_{0}^{\prime \prime}, \hat{Z}_{0, \mathrm{ec}}$, and $\hat{L}_{0}$ reported in Table I. Figures 5(c) and 5(d) show $\left|R^{\perp}\right|,\left|R^{\perp}\right|$, and the reflectance using the existing method $\left|R^{\prime} \angle\right|$ [Eq. (3), Nørgaard et al. (2017a) which does not account for the oblique ear-probe insertion], and the corresponding reflectance group delays $\tau_{\mathrm{g}}^{\perp}, \tau_{\mathrm{g}}^{\llcorner}$, and $\tau_{\mathrm{g}}^{\prime<}$ [Eq. (15)], respectively. Most of the undesired behavior due to the horn loading in $R^{\prime<}$ has been removed in $R^{\perp}$, which is now largely similar to $R^{\perp}$. The small discrepancies, especially visible as a slightly oscillating behavior in $\tau_{\mathrm{g}}^{<}$, may be due to an inaccuracy in $\hat{L}_{0}$ or simply due to an inertance not being an exact representation of the imaginary part of the incident impedance $Z_{\text {meas }, 0}$, though in this case these variations are small compared to those in $\tau_{\mathrm{g}}^{\prime<}$. Figure 5(e) shows the normalized real and imaginary parts of $\hat{Z}_{\text {meas }, 0}^{\perp}$ and $\hat{Z}_{\text {meas }, 0}^{\perp}$ [Eq. (26)], which exhibit a behavior similar to the measured incident impedances of the anechoic waveguide in Fig. 3(a), and Fig. 5(f) shows the normalized estimated plane-wave impedance magnitudes $\left|\hat{Z}_{\mathrm{pw}}^{\perp}\right|$ and $\left|\hat{Z}_{\mathrm{pw}}^{\perp}\right|$ [Eq. (28)] and measured impedance magnitude $\left|Z_{\text {meas }}^{\perp}\right|$ [Eq. (29)]. The slight translation between the extrema in $\hat{Z}_{\mathrm{pw}}^{\perp}$ and $\hat{Z}_{\mathrm{pw}}^{\perp}$ may be due to a difference in insertion depth but the two curves otherwise show substantially the same behavior. In contrast, $Z_{\text {meas }}^{<}$has significantly translated minima due to the total inertance $L_{0}[\mathrm{Eq}$. (8)], and experiences an offset toward higher frequencies corresponding to the ratio $\operatorname{Re}\left\{\hat{Z}_{\text {meas }, 0}^{\perp}\right\} / Z_{0}$.

\section{B. Ear canal}

To exemplify the applicability of the proposed method under realistic conditions, we measured the impedances of 
(a)

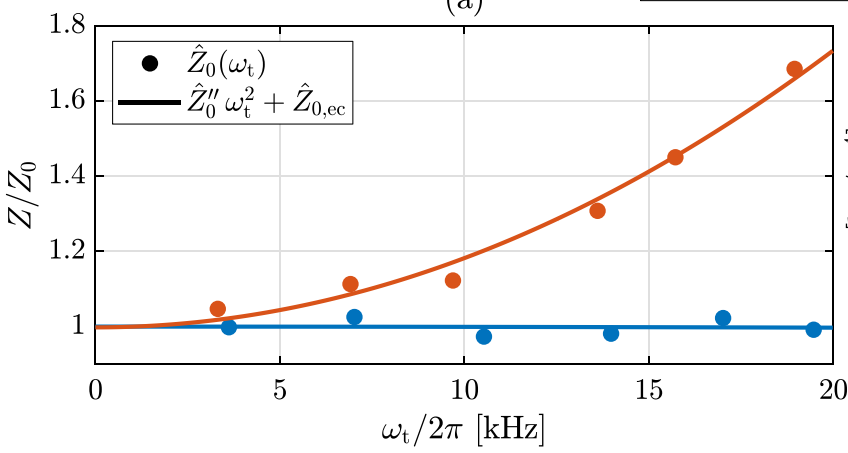

(c)

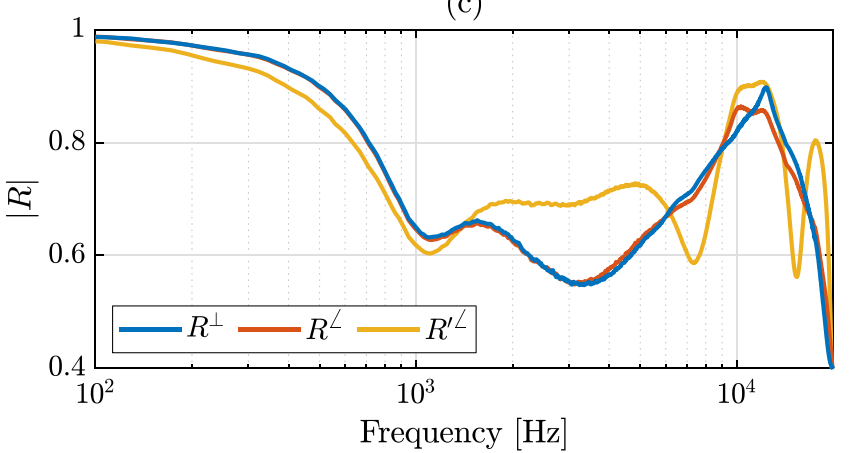

(e)

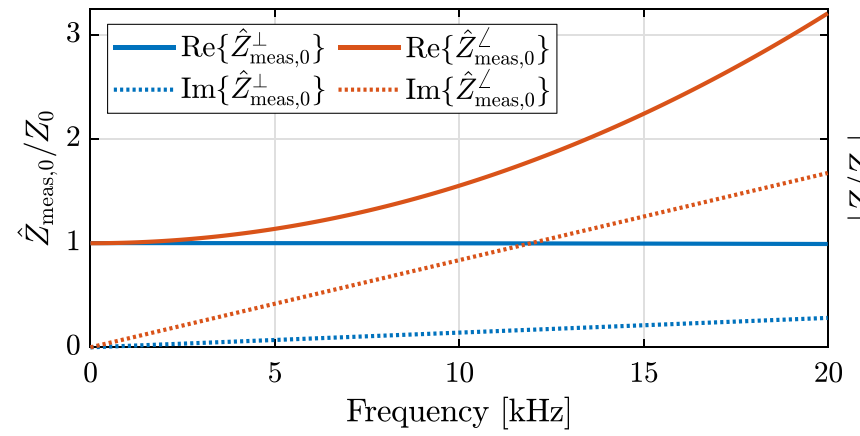

(b)

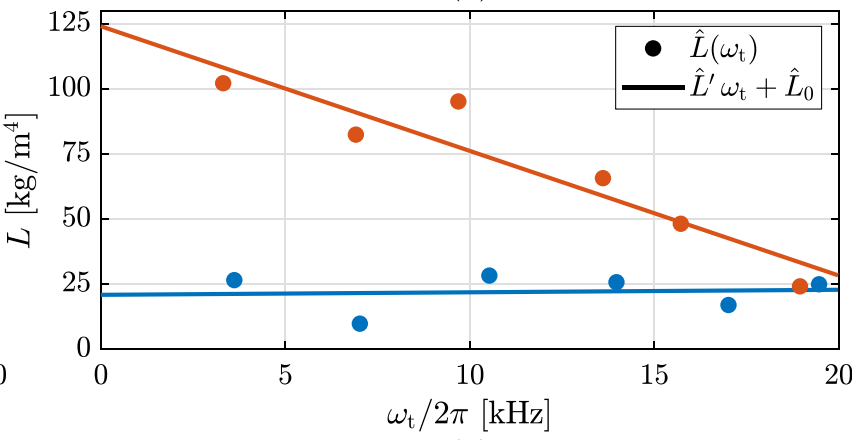

(d)

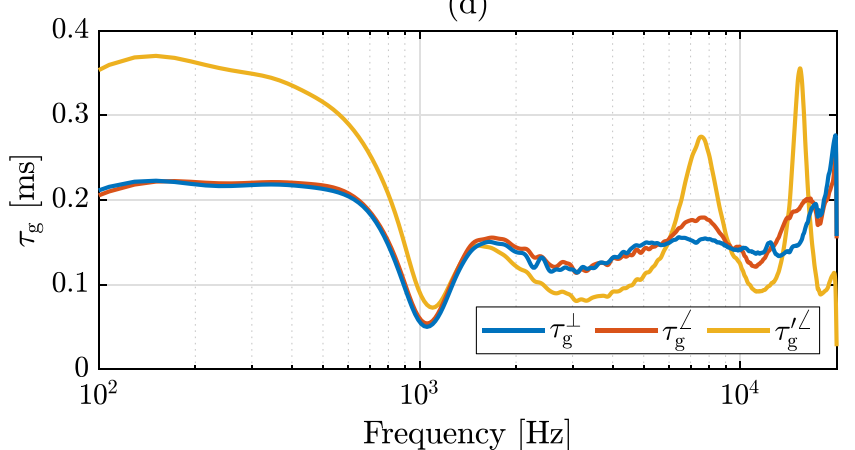

(f)

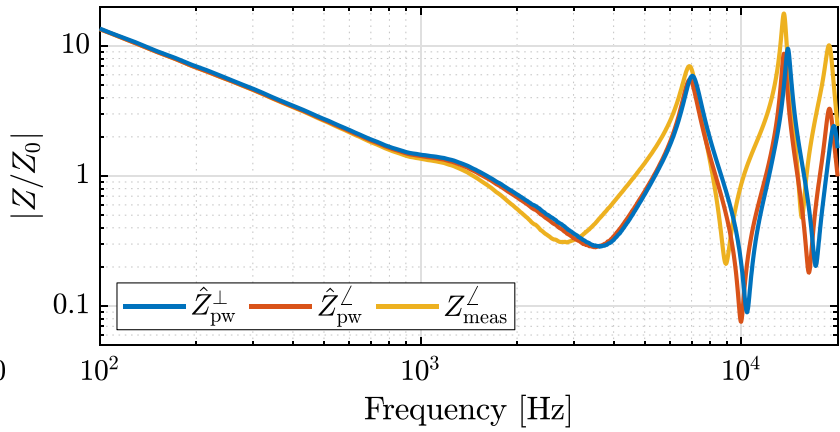

FIG. 5. (Color online) Results for the perpendicular $\perp$ and oblique $\angle$ ear-probe insertions into the occluded-ear simulator. (a),(b) The estimated normalized characteristic impedances $\hat{Z}_{0}\left(\omega_{\mathrm{t}}\right)$ and inertances $\hat{L}\left(\omega_{\mathrm{t}}\right)$ (Nørgaard et al., 2017a) using the truncation frequencies $\omega_{\mathrm{t}}$, and fitted polynomials $\hat{Z}_{0}^{\prime \prime} \omega_{\mathrm{t}}^{2}+\hat{Z}_{0, \mathrm{ec}}[$ Eq. (21)] and $\hat{L}^{\prime} \omega_{\mathrm{t}}+\hat{L}_{0}$ [Eq. (25)], respectively. The (c) reflectance magnitudes $\left|R^{\perp}\right|,\left|R^{\perp}\right|$ [Eq. (27)], and $\left|R^{\prime<}\right|$ [Eq. (3)], (d) corresponding group delays $\tau_{\mathrm{g}}^{\perp}, \tau_{\mathrm{g}}^{<}$, and $\tau_{\mathrm{g}}^{\prime<}$ [Eq. (15)], and (e) normalized estimated incident impedances $\hat{Z}_{\text {meas }, 0}^{\perp}$ and $\hat{Z}_{\text {meas }, 0}^{\perp}$ [Eq. (26)]. (f) The normalized estimated plane-wave impedance magnitudes $\left|\hat{Z}_{\mathrm{pw}}^{\perp}\right|$ and $\left|\hat{Z}_{\mathrm{pw}}^{\perp}\right|$ [Eq. (28)], and measured impedance $\left|Z_{\text {meas }}^{\perp}\right|$ [Eq. (29)].

the ear canal at three different insertion depths: deep, medium, and shallow. Figures 6(a) and 6(b) show the estimated quantities $\hat{Z}_{0}\left(\omega_{\mathrm{t}}\right)$ and $\hat{L}\left(\omega_{\mathrm{t}}\right)$ (Nørgaard et al., 2017a) and the corresponding fitted polynomials $\hat{Z}_{0}^{\prime \prime} \omega_{\mathrm{t}}^{2}+\hat{Z}_{0, \mathrm{ec}}[\mathrm{Eq}$.
(21)] and $\hat{L}^{\prime} \omega_{\mathrm{t}}+\hat{L}_{0}$ [Eq. (25)] for each insertion depth. Table I further lists the quantities characterizing the polynomials. The results indicate that oblique ear-probe insertions may be a substantial problem in ear canals and the degree of

TABLE I. The estimated quantities describing the second-order polynomial $\hat{Z}_{0}^{\prime \prime} \omega_{\mathrm{t}}^{2}+\hat{Z}_{0, \mathrm{ec}}$ [Eq. (21)] and the first-order polynomial $\hat{L}^{\prime} \omega_{\mathrm{t}}+\hat{L}_{0}[\mathrm{Eq} .(25)]$ for the perpendicular and oblique insertions of the ear probe into the occluded-ear simulator (labeled OES in the table) with the known characteristic impedance $Z_{0}$ [Eq. (1)], and using the deep, medium, and shallow insertions into the ear canal. $\hat{Z}_{0}^{\prime \prime}$ and $\hat{L}^{\prime}$ are here multiplied by $\omega_{\mathrm{m}}^{2}$ and $\omega_{\mathrm{m}}$, respectively, to illustrate the contribution at the maximum frequency $\omega_{\mathrm{m}} / 2 \pi=20 \mathrm{kHz}$.

\begin{tabular}{|c|c|c|c|c|c|c|}
\hline & Insertion & $Z_{0}\left[\mathrm{~Pa} \mathrm{~s} / \mathrm{m}^{3}\right]$ & $\hat{Z}_{0}^{\prime \prime} \omega_{\mathrm{m}}^{2}\left[\mathrm{~Pa} \mathrm{~s} / \mathrm{m}^{3}\right]$ & $\hat{Z}_{0, \mathrm{ec}}\left[\mathrm{Pa} \mathrm{s} / \mathrm{m}^{3}\right]$ & $\hat{L}^{\prime} \omega_{\mathrm{m}}\left[\mathrm{kg} / \mathrm{m}^{4}\right]$ & $\overline{\hat{L}_{0}\left[\mathrm{~kg} / \mathrm{m}^{4}\right]}$ \\
\hline \multirow[t]{2}{*}{ OES } & Perpendicular & $9.31 \times 10^{6}$ & $-2.66 \times 10^{4}$ & $9.31 \times 10^{6}$ & 1.9 & 20.9 \\
\hline & Oblique & & $6.85 \times 10^{6}$ & $9.30 \times 10^{6}$ & -95.6 & 124.0 \\
\hline \multirow[t]{3}{*}{ Ear canal } & Deep & $\mathrm{n} / \mathrm{a}$ & $1.93 \times 10^{6}$ & $6.83 \times 10^{6}$ & -25.3 & 94.9 \\
\hline & Medium & & $8.24 \times 10^{6}$ & $6.79 \times 10^{6}$ & -148.8 & 193.4 \\
\hline & Shallow & & $1.19 \times 10^{7}$ & $7.15 \times 10^{6}$ & -236.3 & 232.3 \\
\hline
\end{tabular}




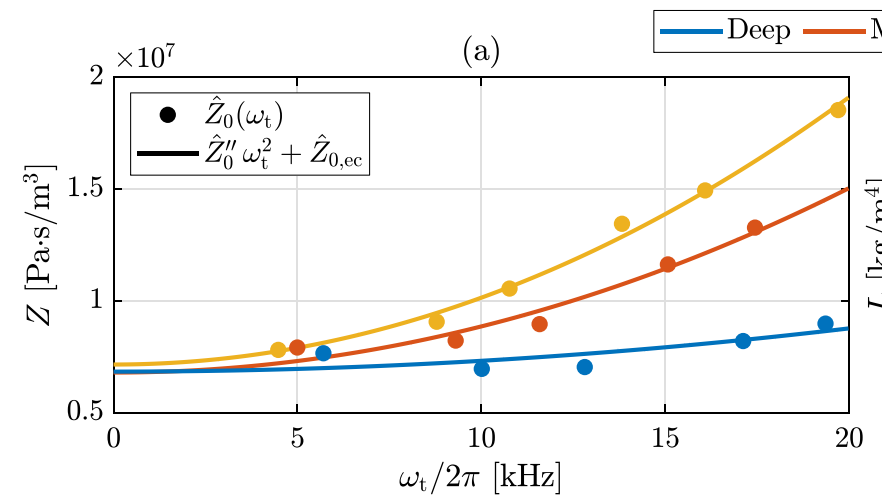

(c)

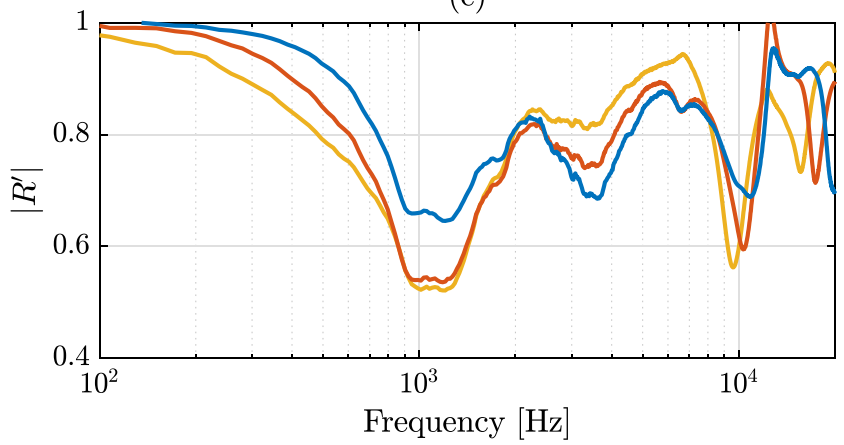

(e)

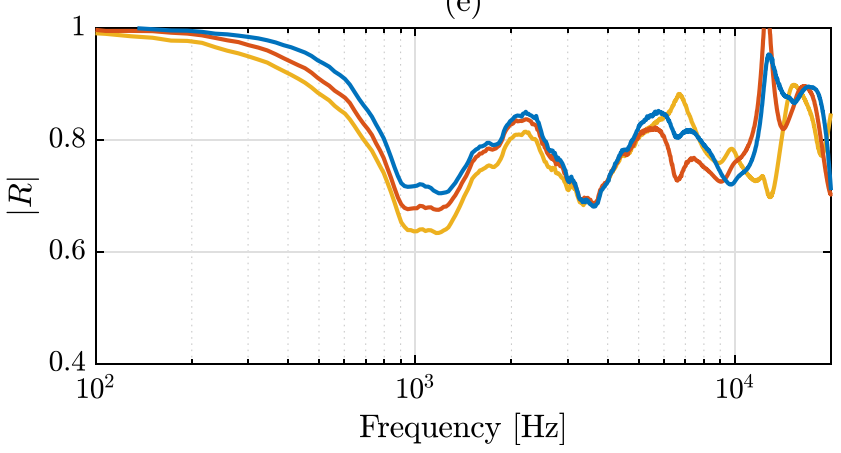

(b)

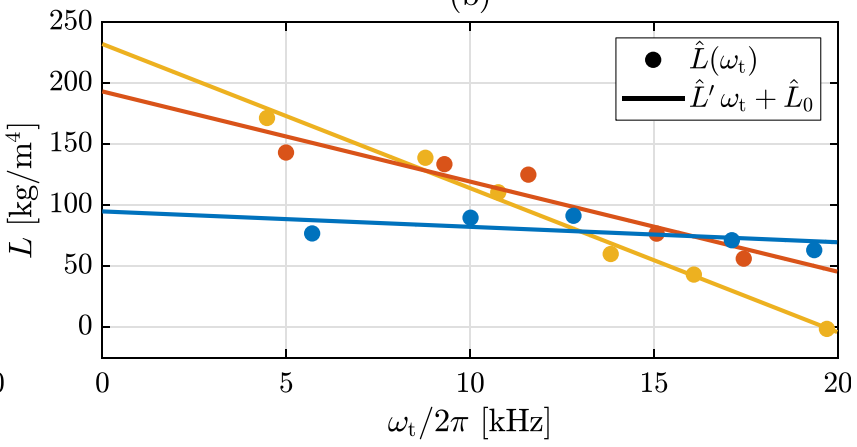

(d)

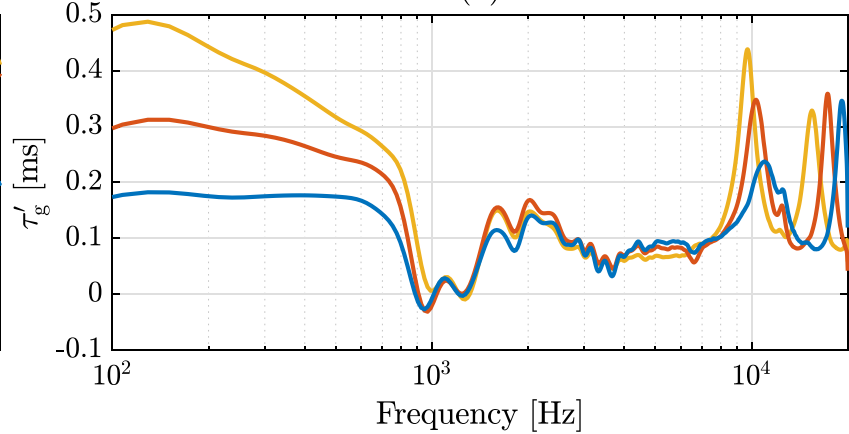

(f)

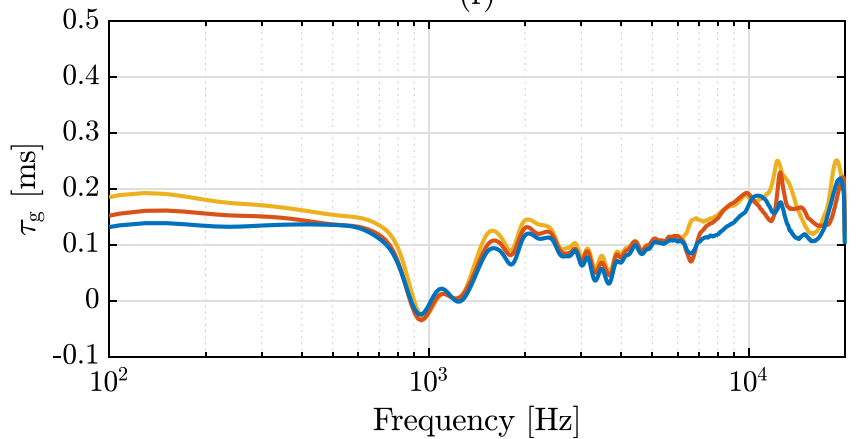

FIG. 6. (Color online) Results for the deep, medium, and shallow insertions of the ear probe into the ear canal. (a), (b) The estimated characteristic impedances $\hat{Z}_{0}\left(\omega_{\mathrm{t}}\right)$ and inertances $\hat{L}\left(\omega_{\mathrm{t}}\right)$ (Nørgaard et al., 2017a), using the truncation frequencies $\omega_{\mathrm{t}}$, and fitted polynomials $\hat{Z}_{0}^{\prime \prime} \omega_{\mathrm{t}}^{2}+\hat{Z}_{0, \mathrm{ec}}[\mathrm{Eq}$. $(21)]$ and $\hat{L}^{\prime} \omega_{\mathrm{t}}+\hat{L}_{0}[\mathrm{Eq}$. (25)], respectively. (c), (d) The reflectance magnitudes $\left|R^{\prime}\right|$ [Eq. (3)] and corresponding group delays $\tau_{g}^{\prime}$ [Eq. (15)], respectively, using the existing method (Nørgaard et al., 2017a), and (e), (f) the reflectance magnitudes $|R|$ [Eq. (27)] and corresponding group delays $\tau_{g}$, respectively, using the proposed method.

obliqueness vary significantly even within the same ear canal at different insertion depths, limiting the ability to estimate the ear-canal characteristic impedance. However, the fitted polynomials represent the data well, suggesting that compensation for the oblique ear-probe insertions may be possible. Figures 6(c) and 6(d) show the reflectance magnitudes $\left|R^{\prime}\right|$ [Eq. (3)] and corresponding group delays $\tau_{g}^{\prime}$ [Eq. (15)], respectively, for the three insertion depths into the ear canal obtained with the existing method (Nørgaard et al., 2017a), and reveal a substantial variation in reflectance similar to that in Fig. 1. Finally, Figs. 6(e) and 6(f) show the obtained reflectance magnitudes $|R|$ [Eq. (27)] and corresponding group delays $\tau_{g}$, respectively, using the proposed method. The results demonstrate a substantial decrease in the dependency of the reflectance on the insertion depth across the entire frequency range. However, we cannot assert whether the effects observed here are caused by actual oblique orientations of the ear probe or something else because we do not directly control the insertion angle. Below $2 \mathrm{kHz}$, a slight variation between the three cases remains which may be caused by non-uniformities in the ear canal or small leaks in the ear-probe fit. The variations in $R$ at 6.5 and $12 \mathrm{kHz}$ coincide with resonance frequencies in the ear probe and we believe they are caused by small shifts in these resonances with the different insertions, perhaps due to small amounts of cerumen or debris entering the probe tube.

\section{DISCUSSION}

Underlying assumptions in the proposed method of compensating for an oblique ear-probe insertion are that the waveguide is uniform and terminated by some impedance discontinuity that results in a substantial reflection, and that the wavelength is much longer than the equivalent horn length, $\lambda \gg l_{\text {horn }}$. Under these conditions and by employing the method of Nørgaard et al. (2017a) at multiple truncation frequencies, we noted that the estimated characteristic impedances and inertances can be used to estimate the incident 
impedance of the horn loading. The simple polynomials fitted to the estimated characteristic impedances and inertances may be considered as merely examples of possible functions to fit to these quantities. They were chosen as the two included parameters in each polynomial provide intuitive information on the ordinate intercept, and concavity or slope. In addition, the polynomials approximate with reasonable accuracy the behavior of the incident impedance of the horn loading in a uniform waveguide within the considered frequency range. With five or six available truncation frequencies for the presented cases, a more detailed model could be at risk of overfitting. In some ears, a smaller number of truncation frequencies may be available, potentially decreasing the robustness of the method and its susceptibility to errors in the various estimates of characteristic impedances and inertances.

Variations in the estimated characteristic impedances and inertances causing deviations from the fitted polynomials may be caused by non-uniformities in the acoustic load when $\lambda \gg l_{\text {horn }}$ is no longer satisfied. The occluded-ear simulator has a built-in protection grid, which introduces a non-uniformity approximately half-way toward its termination from the ear probe. This may explain some of the deviations in the estimated quantities in Figs. 5(a) and 5(b), particularly at lower frequencies. In general, each truncation frequency can be seen as a Nyquist frequency used to synthesize the time-domain transfer functions from which the characteristic impedance and inertance are estimated. Due to the finite speed of sound, the samples in the synthesized time-domain impedance $z(t \leq 0)$ using a gradually decreasing truncation frequency contain gradually more spatial information about the acoustic load as seen from the ear probe. This causes the estimated characteristic impedance to converge to the characteristic impedance of a uniform load toward low truncation frequencies. Despite effects of nonuniformities on the estimated quantities, the fitted polynomials force the estimated incident impedance to exhibit a behavior corresponding to the case where $\lambda \gg l_{\text {horn }}$. For the measurements conducted in this study, these deviations in estimated quantities appear to be negligible.

The proposed method does not take into account the frequency-dependent phase shift in the incident transfer impedance of the horn loading. Time-domain analysis of the ear-canal reflectance using the inverse Fourier transform may therefore be limited because responses resulting from various features may be delayed differently in time.

The proposed method resulted in similar reflectances in a uniform occluded-ear simulator using perpendicular and oblique ear-probe insertions. However, in non-uniform waveguides it may not be possible to adequately distinguish these non-uniformities from the oblique ear-probe insertion using the proposed method. The reported results suggest that obtaining similar estimates of the characteristic impedance across all possible truncation frequencies may be infeasible for typical ear-probe insertions into ear canals. The estimated characteristic impedances and inertances in the ear canal appear to follow a similar pattern as in the uniform occluded-ear simulator, suggesting that the proposed method may be applicable in ear canals. In addition, a decreased dependency of the reflectance on insertion depth in the ear canal was observed. However, this decreased dependency cannot serve as a validation of the accuracy of the obtained ear-canal reflectance because a reliable reference does not exist.

Disregarding non-uniformities in the ear canal, placing the ear probe at the position of a bend may cause the horn loading to appear longer, thereby decreasing the frequency where $\lambda \gg$ $l_{\text {horn }}$ is satisfied. In such a case, a more detailed representation of the incident impedance may be required to obtain an accurate ear-canal reflectance. It is currently uncertain if the seemingly oblique ear-probe insertions in the ear canal are caused by misalignments of the ear-probe orientation relative to the ear-canal walls, placements of the ear probe at ear-canal bends, or different degrees of non-uniformities of the ear canal at the three insertion depths. However, such non-uniformities do seem to pose a significant problem in ear-canal reflectance measurements that need to be accounted for when estimating the characteristic impedance in situ. The proposed method substantially reduced the problem but designing better ear tips to enable a more precise alignment of the ear probe could also be a viable approach.

\section{CONCLUSION}

Estimating the characteristic impedance is a crucial part of ear-canal reflectance measurements. In this paper, we have shown that an oblique ear-probe insertion results in large errors in the measured ear-canal reflectance and prevents accurately estimating the characteristic impedance of the ear canal in situ. These errors could have a detrimental effect on the capability of the ear-canal reflectance in identifying conductive hearing disorders, calibrating stimulus levels, and estimating the emitted pressure above frequencies as low as $2 \mathrm{kHz}$. It has further been demonstrated how the effects of an oblique ear-probe insertion can be represented as a horn loading in front of the ear probe and compensated for in a uniform waveguide by estimating the incident impedance, i.e., were the uniform waveguide anechoic, and substituting it in place of the characteristic impedance when calculating the reflectance. Finally, the proposed method was able to compensate for an oblique ear-probe insertion into an occluded-ear simulator and resulted in a decreased dependency of the ear-canal reflectance on insertion depth in an ear canal. The proposed method could substantially increase the reproducibility and accuracy of ear-canal reflectance measurements, particularly at higher frequencies, however, a systematic assessment in ear canals has yet to be conducted.

\section{ACKNOWLEDGMENTS}

This research was funded by Interacoustics $\mathrm{A} / \mathrm{S}$ and the Innovation Fund Denmark, Grant. No. 5189-00100B.

Allen, J. B. (1986). "Measurement of eardrum acoustic impedance," in Peripheral Auditory Mechanisms, edited by J. Allen, J. Hall, A. Hubbard, S. Neely, and A. Tubis (Springer-Verlag, New York), pp. 44-51.

Benade, A. H. (1988). "Equivalent circuits for conical waveguides," J. Acoust. Soc. Am. 83, 1764-1769. 
Brass, D., and Locke, A. (1997). "The effect of the evanescent wave upon acoustic measurements in the human ear canal," J. Acoust. Soc. Am. 101, 2164-2175.

Caussé, R., Kergomard, J., and Lurton, X. (1984). "Input impedance of brass musical instruments-Comparison between experiment and numerical models," J. Acoust. Soc. Am. 75, 241-254.

Charaziak, K. K., and Shera, C. A. (2017). "Compensating for ear-canal acoustics when measuring otoacoustic emissions," J. Acoust. Soc. Am. 141, 515-531.

Ellison, J. C., Gorga, M., Cohn, E., Fitzpatrick, D., Sanford, C. A., and Keefe, D. H. (2012). "Wideband acoustic transfer functions predict middle-ear effusion," Laryngoscope 122, 887-894.

Feeney, M. P., and Keefe, D. H. (2001). "Estimating the acoustic reflex threshold from wideband measures of reflectance, admittance, and power," Ear Hear. 22, 316-332.

Fletcher, N. H., Smith, J., Tarnopolsky, A. Z., and Wolfe, J. (2005). "Acoustic impedance measurements-Correction for probe geometry mismatch," J. Acoust. Soc. Am. 117, 2889-2895.

Humphrey, R. (2014). www.playrec.co.uk (Last viewed 5 June 2019).

Keefe, D. H., and Benade, A. H. (1981). "Impedance measurement source and microphone proximity effects," J. Acoust. Soc. Am. 69, 1489-1495.

Keefe, D. H., Folsom, R. C., Gorga, M. P., Vohr, B. R., Bulen, J. C., and Norton, S. J. (2000). "Identification of neonatal hearing impairment: Earcanal measurements of acoustic admittance and reflectance in neonates," Ear Hear. 21, 443-461.

Keefe, D. H., Ling, R., and Bulen, J. C. (1992). "Method to measure acoustic impedance and reflection coefficient," J. Acoust. Soc. Am. 91, 470-485.

Keefe, D. H., Sanford, C. A., Ellison, J. C., Fitzpatrick, D. F., and Gorga, M P. (2012). "Wideband aural acoustic absorbance predicts conductive hearing loss in children," Int. J. Audiol. 51, 880-891.

Lewis, J. D. (2018). "The area discontinuity between probe and ear canal as a source of power-reflectance measurement-location variability," J. Acoust. Soc. Am. 143, 1106-1116.

Lewis, J. D., McCreery, R. W., Neely, S. T., and Stelmachowicz, P. G. (2009). "Comparison of in-situ calibration methods for quantifying input to the middle ear," J. Acoust. Soc. Am. 126, 3114-3124.

McCreery, R. W., Pittman, A., Lewis, J., Neely, S. T., and Stelmachowicz, P. G. (2009). "Use of forward pressure level to minimize the influence of acoustic standing waves during probe-microphone hearing-aid verification," J. Acoust. Soc. Am. 126, 15-24.

Merchant, G. R., Röösli, C., Niesten, M. E. F., Hamade, M. A., Lee, D. J., McKinnon, M. L., Ulku, C. H., Rosowski, J. J., Merchant, S. N., and Nakajima, H. H. (2015). "Power reflectance as a screening tool for the diagnosis of superior semicircular canal dehiscence," Otol. Otolaryngol. 36, 172-177.

Nørgaard, K. R., Fernandez-Grande, E., and Laugesen, S. (2017a). "Compensating for evanescent modes and estimating characteristic impedance in waveguide acoustic impedance measurements," J. Acoust. Soc. Am. 142, 3497-3509.

Nørgaard, K. R., Fernandez-Grande, E., and Laugesen, S. (2017b). "Incorporating evanescent modes and flow losses into reference impedances in acoustic Thévenin calibration," J. Acoust. Soc. Am. 142, 3013-3024.

Nørgaard, K. R., Neely, S. T., and Rasetshwane, D. M. (2018). "Quantifying undesired parallel components in Thévenin-equivalent acoustic source parameters," J. Acoust. Soc. Am. 143, 1491-1503.

Piskorski, P., Keefe, D. H., Simmons, J. L., and Gorga, M. P. (1999). "Prediction of conductive hearing loss based on acoustic ear-canal response using a multivariate clinical decision theory," J. Acoust. Soc. Am. 105, 1749-1764.

Rasetshwane, D. M., and Neely, S. T. (2011). "Inverse solution of ear-canal area function from reflectance," J. Acoust. Soc. Am. 130, 3873-3881.

Scheperle, R. A., Goodman, S. S., and Neely, S. T. (2011). "Further assessment of forward pressure level for in situ calibration," J. Acoust. Soc. Am. 130, 3882-3892.

Scheperle, R. A., Neely, S. T., Kopun, J. G., and Gorga, M. P. (2008). "Influence of in situ, sound-level calibration on distortion-product otoacoustic emission variability," J. Acoust. Soc. Am. 124, 288-300.

Souza, N. N., Dhar, S., Neely, S. T., and Siegel, J. H. (2014). "Comparison of nine methods to estimate ear-canal stimulus levels," J. Acoust. Soc. Am. 136, 1768-1787.

Voss, S. E., and Allen, J. B. (1994). "Measurement of acoustic impedance and reflectance in the human ear canal," J. Acoust. Soc. Am. 95, 372-384.

Withnell, R. H., Jeng, P. S., Waldvogel, K., Morgenstein, K., and Allen, J. B. (2009). "An in situ calibration for hearing thresholds," J. Acoust. Soc. Am. 125, 1605-1611. 